\title{
Lysophosphatidic acid protects against acetaminophen-induced acute liver injury
}

\author{
Geon Ho Bae ${ }^{1}$, Sung Kyun Lee ${ }^{1}$, Hyung Sik Kim ${ }^{1}$, Mingyu Lee ${ }^{2}$, Ha Young Lee ${ }^{1}$ and Yoe-Sik Bae ${ }^{1,2}$
}

We investigated the effect of lysophosphatidic acid (LPA) in experimental acetaminophen (APAP)-induced acute liver injury. LPA administration significantly reduced APAP-challenged acute liver injury, showing attenuated liver damage, liver cell death and aspartate aminotransferase and alanine aminotransferase levels. APAP overdose-induced mortality was also significantly decreased by LPA administration. Regarding the mechanism involved in LPA-induced protection against acute liver injury, LPA administration significantly increased the glutathione level, which was markedly decreased in APAP challenge-induced acute liver injury. LPA administration also strongly blocked the APAP challenge-elicited phosphorylation of JNK, ERK and GSK3 $\beta$, which are involved in the pathogenesis of acute liver injury. Furthermore, LPA administration decreased the production of TNF- $\alpha$ and IL-1 $\beta$ in an experimental drug-induced liver injury animal model. Mouse primary hepatocytes express $\mathrm{LPA}_{1,3-6}$, and injection of the LPA receptor antagonist KI16425 (an LPA 1,3 -selective inhibitor) or H2L 5765834 (an $\mathrm{LPA}_{1,3,5}$-selective inhibitor) did not reverse the LPA-induced protective effects against acute liver injury. The therapeutic administration of LPA also blocked APAPinduced liver damage, leading to an increased survival rate. Collectively, these results indicate that the well-known bioactive lipid LPA can block the pathogenesis of APAP-induced acute liver injury by increasing the glutathione level but decreasing inflammatory cytokines in an $\mathrm{LPA}_{1,3,5}$-independent manner. Our results suggest that LPA might be an important therapeutic agent for drug-induced liver injury.

Experimental \& Molecular Medicine (2017) 49, e407; doi:10.1038/emm.2017.203; published online 8 December 2017

\section{INTRODUCTION}

Drug-induced acute liver injury, the major cause of acute liver failure in the United States and European countries, is mainly caused by overdose of acetaminophen (APAP). ${ }^{1}$ APAP-induced acute liver injury is accompanied by serious hepatotoxicity and hepatocellular necrosis due to an accumulation of $\mathrm{N}$-acetyl-pbenzoquinone-imine (NAPQI), a toxic metabolite of APAP. ${ }^{2}$ NAPQI forms a covalent bond with intracellular proteins, including the antioxidant glutathione (GSH), and impairs the function of mitochondria, resulting in hepatotoxicity and hepatocyte necrosis. ${ }^{3}$ In the early phase of liver injury, an accumulation of reactive oxygen species (ROS) occurs, which causes the activation of GSK3 $\beta$, MKK4/7 and ERK signaling axes. These signaling axes can lead to JNK phosphorylation, which is a major pathway for drug-induced liver injury that leads to hepatocyte death. ${ }^{4}$ Liver injury-induced inflammatory mediators and damage-associated molecular patterns can also mediate hepatotoxicity. ${ }^{5}$ Previous studies have demonstrated that some chemokine/chemoattractant receptors, such as
CXCR2 and formyl peptide receptor (FPR)1, can mediate the pathogenic response of acute liver injury caused by APAP overdose. ${ }^{6}$ The liver injury-induced production of chemokines (CXCL1 and CXCL2) and mitochondria-derived formyl peptides can stimulate CXCR2 and FPR1, respectively. ${ }^{6}$ The liver damage-induced release of mitochondrial DNA also mediates hepatotoxicity via TLR9. ${ }^{6}$ Although some endogenous molecules that mediate acute liver injury have been identified, studies of additional important endogenous molecules that protect against acute liver injury are limited.

Lysophosphatidic acid (LPA) is a well-known lipid-derived ligand that can stimulate various cell types. ${ }^{7}$ Previous studies have demonstrated that LPA can regulate cell growth, cell differentiation and cellular motility. ${ }^{8,9}$ LPA also plays a crucial role in the metastasis and invasion of tumor cells, and mediates tumor progress. ${ }^{10}$ Regarding the cellular targets of LPA, several G-protein-coupled receptors (GPCRs), such as $\mathrm{LPA}_{1-6}$, have been reported to bind to LPA and mediate LPA-induced pathophysiological responses. ${ }^{11}$ LPA receptors, especially

\footnotetext{
${ }^{1}$ Department of Biological Sciences, Sungkyunkwan University, Suwon, Republic of Korea and ${ }^{2}$ Department of Health Sciences and Technology, Samsung Advanced Institute for Health Sciences and Technology, Seoul, Republic of Korea

Correspondence: Professor Y-S Bae, Department of Biological Sciences, Sungkyunkwan University, 2066 Seobu-ro, Jangan-gu, Suwon 16419, Republic of Korea.

E-mail: yoesik@skku.edu

Received 11 April 2017; revised 1 June 2017; accepted 5 June 2017
} 
$\mathrm{LPA}_{1,3,5,6}$ are also expressed in mouse liver tissue. ${ }^{12-14}$ The stimulation of hepatocytes by LPA can elicit hepatocyte proliferation and prevent apoptosis. ${ }^{15}$ Another study also demonstrated that LPA can inhibit primary hepatocyte proliferation induced by hepatocyte growth factor and epidermal growth factor. ${ }^{16}$ Previously, Watanabe et al. ${ }^{17}$ demonstrated that carbon tetrachloride-induced liver injury can increase plasma LPA levels. Autotaxin is an enzyme involved in the generation of LPA. Auotaxin level is also significantly increased in dimethylnitrosamine-induced acute liver injury. ${ }^{17}$ However, the effect of LPA on acute liver injury remains unclear. Therefore, the objective of this study was to examine the effect of LPA on APAP-induced acute liver damage using an experimental animal model and to investigate the mechanism involved in the LPA-induced therapeutic activity against liver injury.

\section{MATERIALS AND METHODS}

\section{Animal studies}

C57BL/6 mice were purchased from Orient Bio (Seongnam, Korea). All experiments involving animals were approved by the Institutional Review Committee for Animal Care and Use at Sungkyunkwan University (Suwon, Korea). The mice were fasted for $16 \mathrm{~h}$. APAP (Sigma, St Louis, MO, USA) was dissolved in $55-60^{\circ} \mathrm{C}$ warm $1 \times$ PBS, cooled to $37^{\circ} \mathrm{C}$, and administered to the mice by oral gavage (400 $\mathrm{mg} \mathrm{kg}^{-1}$ ). LPA (AVANTI, Alabaster, AL, USA) or NAC was dissolved in PBS with 1\% DMSO or in PBS, respectively, and intraperitoneally injected into the mice $30 \mathrm{~min}$ prior to or 1 or $3 \mathrm{~h}$ after the APAP administration. The LPA receptor antagonists KI16425 (Sigma) and H2L 5765834 (TOCRIS, Minneapolis, MN, USA) were intraperitoneally injected at 30 min prior to the LPA administration.

\section{Histopathology and immunohistochemistry}

After isolating the livers from the APAP-induced acute liver injury models, the livers were fixed with $10 \%$ neutral buffered formalin. The liver tissues were embedded with paraffin, and $5 \mu \mathrm{m}$ sections were cut from them. They were stained with hematoxylin and eosin for histopathological analysis. Fixed liver sections were also used for TUNEL assay after they were incubated with terminal deoxynucleotidyl transferase dUTP nick and labeled with enzyme for $1 \mathrm{~h}$ at $37^{\circ} \mathrm{C}$ in the dark. After the slides were washed three times in PBS for $3 \mathrm{~min}$, they were incubated with POD substrate for $30 \mathrm{~min}$ at $37^{\circ} \mathrm{C}$ in the dark. TUNEL-positive cells were detected using DAB solution (Roche, Basel, Switzerland). Necrotic cells were evaluated using a Leica ICC50 HD microscope (Leica Microsystems, Wetzlar, Germany) with magnification of $\times 200$.

\section{Measurement of serum alanine transaminase (ALT) and aspartate aminotransferase (AST)}

ALT and AST levels were measured using ALT and AST activity assay kits (Sigma) according to the manufacturer's instructions.

\section{Western blot analysis}

After isolating the livers from the APAP-induced acute liver injury model, the livers were homogenized with RIPA buffer $(150 \mathrm{~mm} \mathrm{NaCl}$, Tris-HCl, pH 7.5, SDS 0.1\%, Triton X-100 1\%, EDTA 2 mм, 0.5\% Na-deoxycholate) containing proteinase inhibitor. Proteins were separated by SDS-PAGE and transferred to a PVDF membrane, which was then probed with primary antibodies against phosphorylated JNK, total JNK, phosphorylated ERK, total ERK, phosphorylated GSK3 $\beta$ or total GSK3 $\beta$ (Cell Signaling Technology, Danvers, MA, USA). These membranes were then incubated with anti-rabbit (Enzo Life Sciences Inc., Farmingdale, NY, USA) or anti-mouse (KPL Inc., Gaithersburg, MD, USA) secondary antibodies.

\section{GSH measurement}

Liver tissues $(60 \mathrm{mg}$ ) from APAP-induced acute liver injury mice were homogenized with $5 \%$ metaphosphoric acid ( $10 \mathrm{mg}$ of liver per $500 \mu \mathrm{l}$ of $5 \%$ metaphosphoric acid). Lysates were centrifuged at 12000 r.p.m. for $10 \mathrm{~min}$ at $4{ }^{\circ} \mathrm{C}$. GSH measurement was performed for supernatant using a glutathione (GSSG/GSH) detection kit (Enzo Life Sciences Inc., Farmingdale, NY, USA) according to the manufacturer's instructions. The total GSH level was calculated based on the net slope of the standard curve.

\section{ELISA}

Blood was harvested from the acute liver injury mice at $12 \mathrm{~h}$ postAPAP treatment. After removing cells from the biofluids via centrifugation (12 000 r.p.m. for $1 \mathrm{~min}$ ), the concentrations of the indicated cytokines were determined by ELISA (eBioscience Inc., San Diego, CA, USA) according to the manufacturer's instructions.

\section{Quantitative real-time polymerase chain reaction}

Total RNA was isolated from whole liver lysates or primary hepatocytes using TRIzol according to the manufacturer's instructions (Ambion, Thermo Fisher Scientific, Waltham, MA, USA). One microgram of RNA was reverse-transcribed using the Maxime RT PreMix Kit (Intron, Seongnam, Korea). The cDNA was subjected to QPCR using the Rotor-Gene SYBRGreen PCR Kit (QIAGEN, Hilden, Germany) with gene-specific primers: Gpx-1: forward 5'-CCAGGA GAATGGCAAGAATGA-3', reverse 5'-TCTCACCATTCACTTCGCA CTT-3'; Sod2: forward 5'-TCCCAGACCTGCCTTACGACTAT-3', reverse $5^{\prime}$-GGTGGCGTTGAGATTGTTCA-3'; PGC-1 $\alpha$ : forward 5'-AGCACACGTTTATTCACGGGT-3', reverse 5'-GCCCCCAAGTC CTCACATG-3'; FGF21: forward 5'-AGATCAGGGAGGATGGAACA -3', reverse 5'-CACCCAGGATTTGAATCAGC-3'; LPA1: forward 5'-CCAGGAGGAATCGGGACAC-3', reverse 5'-CAATAACAAGAC CAATCCCGGA-3'; LPA2: forward 5'-GTCAAGACGGTTGTC ATCATTCT-3', reverse 5'-GAAGCATGATCCGCGTGCT-3'; LPA3: forward 5'-CCACTTTCCСТTCTACTACCTGCT-3', reverse 5'-GAC GGTCAACGTTTTCGACACC-3'; LPA4: forward 5'-CAGTGCC TCCCTGTTTGTCTTC-3', reverse $5^{\prime}$-GAGAGGGCCAGGTTGGTG AT-3'; LPA5: forward 5'-TCCACGCTGGCTGTATATGG-3', reverse 5'-TCGCGGTCCTGAATACTGTTC-3'; LPA6: forward 5'-GATCAC TCTCTGCATCGCTGTTTC-3', reverse 5'-CCCTGAACTTCAGA GAACCTGGAG-3' ${ }^{13,18,19}$ Relative gene expression levels were normalized to that of the GAPDH expression level. ${ }^{20}$

\section{Isolation of primary hepatocytes for quantitative real-time polymerase chain reaction}

Primary hepatocytes were isolated from C57BL/6N mice following liver perfusion with $50 \mathrm{ml}$ in a buffer containing $66.7 \mathrm{mM} \mathrm{NaCl}$, $6.7 \mathrm{~mm} \mathrm{KCl}, 50 \mathrm{~mm}$ HEPES and $4.8 \mathrm{~mm} \mathrm{CaCl}_{2} 2 \mathrm{H}_{2} \mathrm{O}$, collagenase type IV after $20 \mathrm{ml}$ of a buffer containing $66.7 \mathrm{~mm} \mathrm{NaCl}, 6.7 \mathrm{~mm} \mathrm{KCl}$, $50 \mathrm{~mm}$ HEPES and $2.5 \mathrm{~mm}$ EGTA. The cells were centrifuged at 500 r.p.m. for $4 \mathrm{~min}$. Primary hepatocytes were separated from dead cells and other cell types by Percoll gradient centrifugation (1250 r.p.m. for $5 \mathrm{~min})$. 

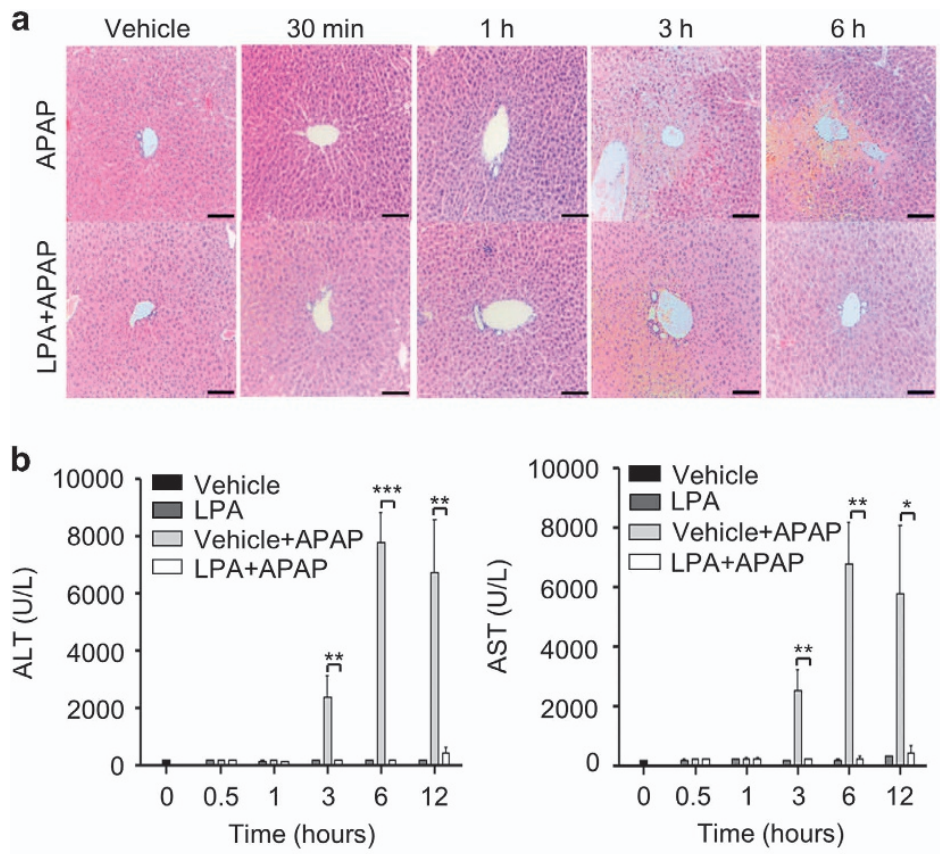

$12 \mathrm{~h}$
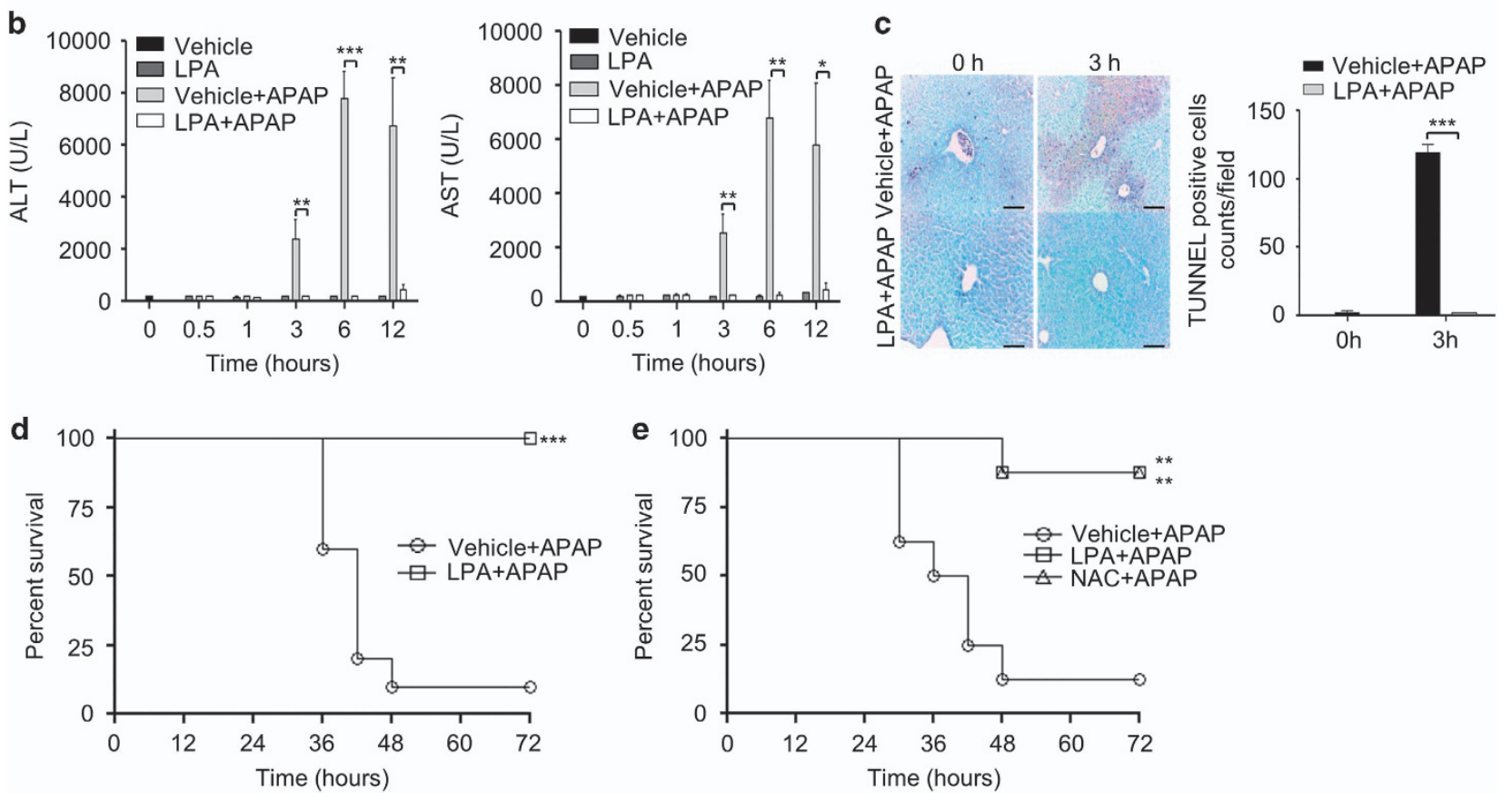

Figure 1 LPA protects against experimental APAP-induced acute liver injury. (a-c) After fasting for $16 \mathrm{~h}$, APAP (400 mg kg-1) was administered to mice. Vehicle (PBS containing 1\% DMSO) or LPA was injected 30 min before the APAP treatment. The mice were killed at $0.5,1,3,6$ and $12 \mathrm{~h}$ after the treatment, and their livers were stained with hematoxylin and eosin (magnification: $\times 200)(a)$. The serum levels of AST and ALT in the mice were measured by enzyme activity assays (b). Necrotic cells were visualized by TUNEL histology (magnification: $\times 200)$ and enumerated $(\mathbf{c})$. (d, e) For the survival rate, the mice were administered APAP $(750 \mathrm{mg} \mathrm{kg}-1)$ at $30 \mathrm{~min}$ after intraperitoneal injection with the vehicle (PBS containing $1 \%$ DMSO), LPA $\left(4 \mathrm{mg} \mathrm{kg}^{-1}\right)$ or NAC (150 mg kg-1). The survival rate was monitored every $6 \mathrm{~h}$ for $72 \mathrm{~h}(\mathbf{d}, \mathbf{e})$. The data are expressed as the mean \pm s.e.m. $\left(n=5\right.$ for $\mathbf{b}, \mathbf{c}$, right). ${ }^{*} P<0.05$; ${ }^{*} P<0.01$; ${ }^{* * *} P<0.001$ by $t$-test. Survival was monitored for $72 \mathrm{~h} .{ }^{* * *} P<0.001$ by ANOVA. Sample size: $n=10$ (d) or 8 (e). The data are representative of two independent experiments with individual samples. Each experiment was performed in quintuplicate. Scale bar, $100 \mu \mathrm{m}(\mathbf{a}, \mathbf{c}$, left).

\section{Statistical analysis}

The results that were collected were evaluated using GraphPad prism software. All of the results are expressed as the mean \pm standard error of the mean (s.e.m.). The statistical analysis was performed using oneway analysis of variance test followed by Student's $t$-test. The survival data were analyzed using the log-rank test and ANOVA. A $P$-value $\leqslant 0.05$ was considered statistically significant.

\section{RESULTS}

\section{LPA administration blocks APAP overdose-induced acute liver injury}

We investigated the effect of LPA administration in APAPinduced acute liver injury. An APAP challenge induced substantial liver damage in a time-dependent manner based on histopathological analysis with hematoxylin and eosin staining of liver sections (Figure 1a). LPA administration markedly blocked liver damage induced by the APAP challenge (Figure 1a). Serum levels of AST and ALT, two well-defined markers of liver damage, were strongly elevated in a timedependent manner in APAP-challenged mice (Figure 1b). AST and ALT showed maximal activities at $6 \mathrm{~h}$ after APAP gavage. However, liver damage-associated enzyme levels were significantly reduced by LPA administration (Figure 1b). LPA administration alone without the APAP challenge did not affect the levels of AST or ALT (Figure 1b). APAP challengeinduced liver damage was also assessed by TUNEL histology. TUNEL-positive cells were strongly decreased after LPA 
a

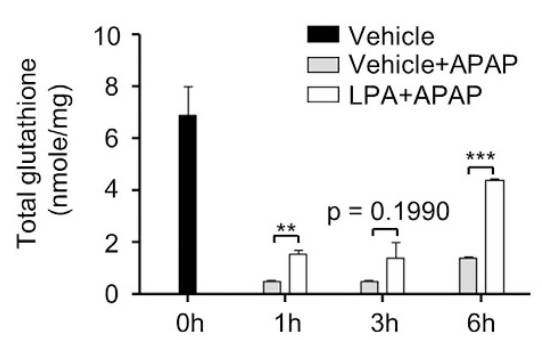

b

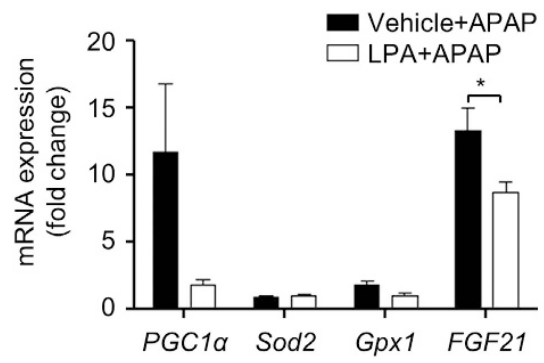

c

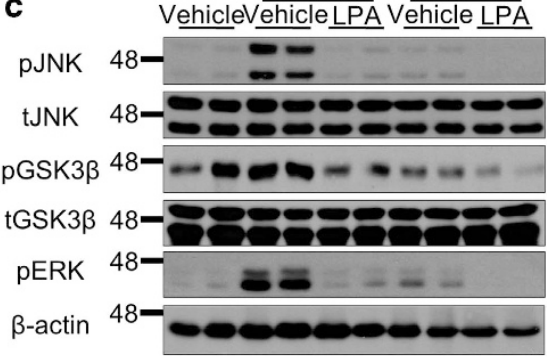

Figure 2 LPA recovers APAP-induced liver depletion of GSH and blocks JNK, ERK, and GSK3 $\beta$ activation. (a-c) The mice were challenged with APAP (400 $\mathrm{mg} \mathrm{kg}^{-1}$ ) or the vehicle control (PBS) by oral gavage at $16 \mathrm{~h}$ after fasting. Either the vehicle (PBS containing $1 \%$ DMSO) or LPA (4 mg kg-1) was administered at $30 \mathrm{~min}$ before the APAP challenge. The mice were killed at $1,3,6$ and $12 \mathrm{~h}$ after the APAP challenge. The total GSH levels in the livers were determined using a commercial GSH assay kit (a). The mice were killed $6 \mathrm{~h}$ after, and the mRNA expression level of the entire liver was investigated (b). The levels of phosphorylated JNK, total JNK, phosphorylated ERK, total ERK, phosphorylated GSK3 $\beta$ and total GSK3 $\beta$ were determined by western blot analysis using whole liver organ lysates (c). The data are expressed as the mean \pm s.e.m. $(n=5$ for $\mathbf{a}, \mathbf{b})$. ${ }^{*} P<0.05$; ${ }^{* *} P<0.01$; ${ }^{* * *} P<0.001$ by $t$-test. The data are representative of three independent experiments (c).
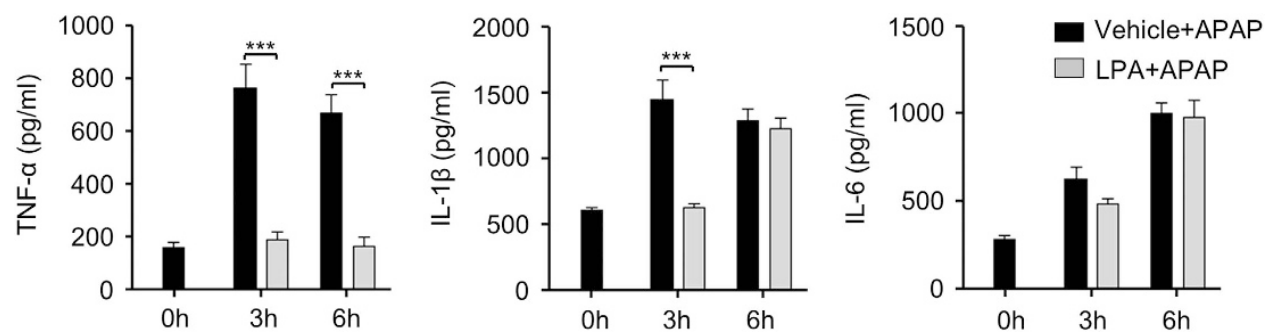

Figure 3 LPA decreases TNF- $\alpha$ and IL-1 $\beta$ levels in APAP-administered mice. The mice were challenged with APAP (400 mg kg-1) or the vehicle control (PBS) by oral gavage after $16 \mathrm{~h}$ of fasting. Either the vehicle (PBS containing $1 \%$ DMSO) or LPA (4 mg kg-1) was administered at 30 min before the APAP challenge. These mice were killed, and the cytokine concentrations in their serum were determined by ELISA. The data are expressed as the mean \pm s.e.m. $(n=5) .{ }^{* *} P<0.001$ by $t$-test.

administration in APAP challenged mice (Figure 1c). We also examined the effect of LPA on APAP-induced mortality. LPA administration significantly protected mice against APAP challenge-induced mortality, showing a survival rate of 100 vs $10 \%$ for the vehicle-administered mice at $72 \mathrm{~h}$ post-APAP challenge (Figure 1d). The only pharmacological option available for APAP overdose patients since the 1970s is $\mathrm{N}$-acetylcysteine (NAC), the precursor of GSH. ${ }^{21}$ Therefore, we compared the effects of LPA on APAP-induced mortality with that of NAC administration. LPA $\left(4 \mathrm{mg} \mathrm{kg}^{-1}\right)$ administration showed the same protective effects as NAC (150 $\mathrm{mg} \mathrm{kg}^{-1}$ ) against APAP-induced mortality (Figure 1e).

LPA enhances the recovery of antioxidant GSH levels and decreases JNK, ERK and GSK3 $\beta$ phosphorylation in the liver following APAP challenge

GSH can detoxify NAPQI to prevent accumulation of APAPprotein adducts and oxidative stress in a liver injury animal model. ${ }^{2}$ GSH depletion is associated with APAP overdoseinduced liver damage in mice. ${ }^{22}$ Liver GSH levels were markedly depleted after the APAP challenge compared to those in the vehicle-challenged mice (at the $0 \mathrm{~h}$ time point). However, liver GSH levels were slightly recovered at $6 \mathrm{~h}$ post APAP (Figure 2a). The administration of LPA significantly enhanced GSH recovery at $1-6 \mathrm{~h}$ in APAP-challenged mice (Figure 2a). We also investigated whether LPA affects the expression of antioxidant genes, including $P G C-1 \alpha$, Sod2, Gpx-1 and FGF21, in the APAP model. LPA administration did not increase the expression of these antioxidant genes (Figure 2b).

Previously, the phosphorylation of JNK and ERK has been reported to be associated with APAP-induced acute liver injury. ${ }^{23}$ In this study, we also observed that APAP challenge strongly elicited JNK and ERK phosphorylation in the liver compared to that of vehicle (PBS) controls based on western blot analysis (Figure 2c). However, APAP-induced JNK and ERK phosphorylation levels were strongly decreased in the livers of mice that were administered LPA (Figure 2b). It has been reported that GSK3 $\beta$ is an upstream molecule of JNK and ERK involved in the pathological process of APAP-induced acute liver injury. ${ }^{4}$ The APAP challenge induced GSK3 $\beta$ phosphorylation, which was markedly attenuated by LPA administration (Figure 2c).

\section{LPA administration decreases TNF- $\alpha$ and IL-1 $\beta$ in experimental drug-induced liver injury}

It has been reported that some inflammatory cytokines, such as TNF- $\alpha$ and IL- $1 \beta$, can mediate the pathological process of 
APAP-induced drug-induced acute liver injury. ${ }^{5}$ Therefore, we examined the effect of LPA administration on the production of these inflammatory cytokines in this study. The APAP overdose challenge strongly increased the production of TNF- $\alpha$ and IL-1 $\beta$ in the APAP acute liver injury model (Figure 3). However, the production of these inflammatory cytokines was significantly decreased by the administration of LPA (Figure 3). The TNF- $\alpha$ level was significantly decreased by LPA administration at $3 \mathrm{~h}$. This inhibitory effect was sustained at $6 \mathrm{~h}$ after the APAP overdose administration. The IL- $1 \beta$ level was significantly decreased by LPA administration at $3 \mathrm{~h}$ but not at $6 \mathrm{~h}$ after APAP administration (Figure 3). Unlike TNF- $\alpha$ or IL-1 $\beta$, the production of IL- 6 , another important proinflammatory cytokine, was not affected by LPA administration (Figure 3).

LPA-induced protective effects against acute liver injury are independently mediated by $\mathrm{LPA}_{1}, \mathrm{LPA}_{3}$ and $\mathrm{LPA}_{5}$

Previously, LPA was reported to be able to bind to several different cell surface GPCRs such as $\mathrm{LPA}_{1-6 .}{ }^{11}$ Other previous reports have demonstrated that $\mathrm{LPA}_{5}$ and a low level of $\mathrm{LPA}_{1}$ are expressed in mouse and human livers. ${ }^{12}$ In this study, we performed q-PCR analysis to investigate which members of the LPA receptor were expressed in primary hepatocytes. We found that mouse primary hepatocytes expressed $\mathrm{LPA}_{1,3-6}$ but not $\mathrm{LPA}_{2}$ (Figure 4a). As we detected the expression of $\mathrm{LPA}_{1}$ and $\mathrm{LPA}_{5}$ in mouse primary hepatocytes, we examined whether LPA could induce a protective effect against APAP overdoseinduced acute liver injury via well-known surface GPCRs using pharmacological inhibitors. KI16425, an $\mathrm{LPA}_{1,3}$-selective inhibitor, and H2L 5765834, an $\mathrm{LPA}_{1,3,5}$-selective inhibitor, failed to block LPA-induced inhibitory effects against acute liver injury (Figure $4 \mathrm{~b}$ ). The two LPA receptor antagonists (KI16425 and H2L 5765834) failed to affect the LPA-induced decrease of ALT in the APAP overdose-induced acute liver injury model (Figure 4b). Moreover, liver histology showed that these LPA receptor antagonists (KI16425 or H2L 5765834) did not reverse the LPA-induced protective effect on liver damage (Figure 4c). These results suggest that LPA-induced protective effects against drug-induced liver injury (DILI) are not mediated by $\mathrm{LPA}_{1}, \mathrm{LPA}_{3}$ or $\mathrm{LPA}_{5}$.

\section{LPA shows therapeutic effects in experimental DILI}

We also examined whether LPA shows therapeutic effects against APAP-induced acute liver injury. To this end, we administered LPA 1 or $3 \mathrm{~h}$ after the APAP challenge and measured liver damage by histopathological analysis with hematoxylin and eosin staining of liver sections. APAPinduced liver damage was strongly blocked by the therapeutic administration of LPA (Figure 5a). Moreover, the therapeutic administration of LPA protected mice against APAP-induced mortality, showing a survival rate of $75 \%$ vs $0 \%$ for vehicleadministered mice at $72 \mathrm{~h}$ post-APAP challenge (Figure $5 \mathrm{~b}$ ).
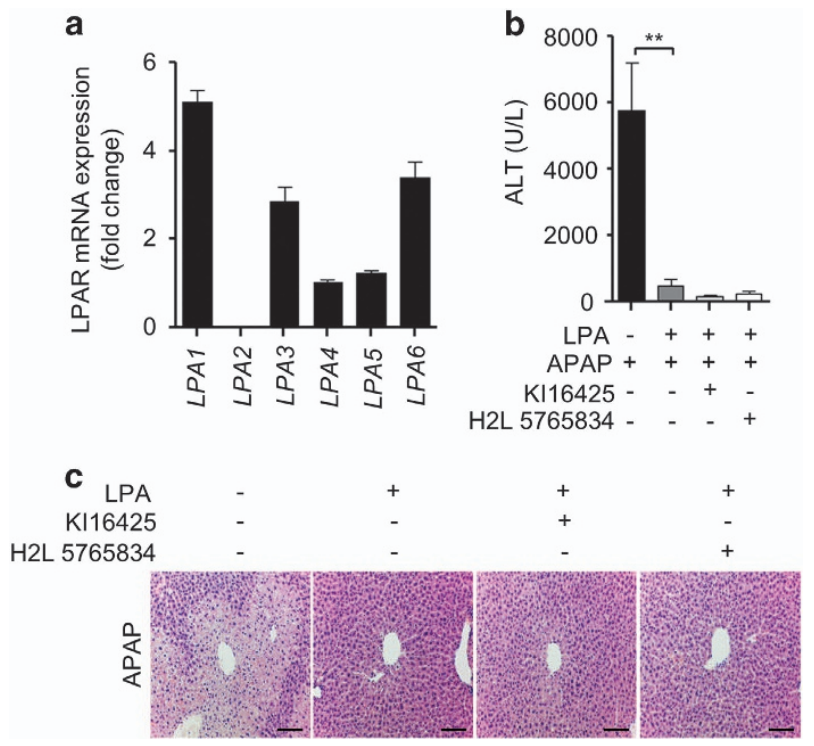

Figure 4 LPA-induced protective effects against experimental APAP-induced acute liver injury is not mediated by $L^{2} A_{1,3}$ or 5 . (ac) APAP (400 mg kg-1) was administered to the mice. Either the vehicle (PBS containing 1\% DMSO) or LPA was injected at 30 min before the APAP treatment. Either the vehicle or LPA receptor antagonists $\left(20 \mathrm{mg} \mathrm{kg}^{-1}\right.$ of $\mathrm{KI} 16425$ or $\mathrm{H} 2 \mathrm{~L} 5765834$ ) were injected at $30 \mathrm{~min}$ before the LPA administration. After $6 \mathrm{~h}$, the mRNA expression levels of LPA receptors were measured by quantitative real-time polymerase chain reaction in primary hepatocytes (a). The levels of ALT in the sera from mice at $6 \mathrm{~h}$ after the APAP challenge were measured by enzyme activity assays (b). The mice were killed $6 \mathrm{~h}$ later, and their livers were stained with hematoxylin and eosin (magnification: $\times 200$ ) (c). The data are expressed as the mean \pm s.e.m. $(n=5$ for $\mathbf{b}) .{ }^{*} P<0.05$ by $t$-test. The data are representative of two independent experiments. Each experiment was performed in quintuplicates (a, b). Scale bar, $100 \mu \mathrm{m}$ (c).

\section{DISCUSSION}

Previously, LPA has been reported to be increased in carbon tetrachloride-induced liver fibrosis and dimethylnitrosamineinduced acute liver injury. ${ }^{17}$ LPA can also stimulate hepatocyte proliferation in culture. ${ }^{16}$ However, the functional role of an increased level of LPA in acute liver injury has not yet been reported. In this study, we demonstrated that administration of bioactive lipid LPA could elicit protective effects in experimental acute liver injury induced by APAP overdose (Figure 1). LPA blocked the APAP administration-induced sustained phosphorylation of JNK via oxidative stress, leading to hepatoprotectivity (Figure 2). LPA also blocked the production of inflammatory cytokines (TNF- $\alpha$ and IL-1 $\beta$ ) during acute liver injury induced by an overdose of APAP (Figure 3). Taken together, previous reports and our current findings suggest that the LPA increase during acute liver injury might have protective effects against acute liver injury to avoid severe liver damage. LPA might act as a negative regulator of acute liver injury to maintain the homeostasis of liver health. 

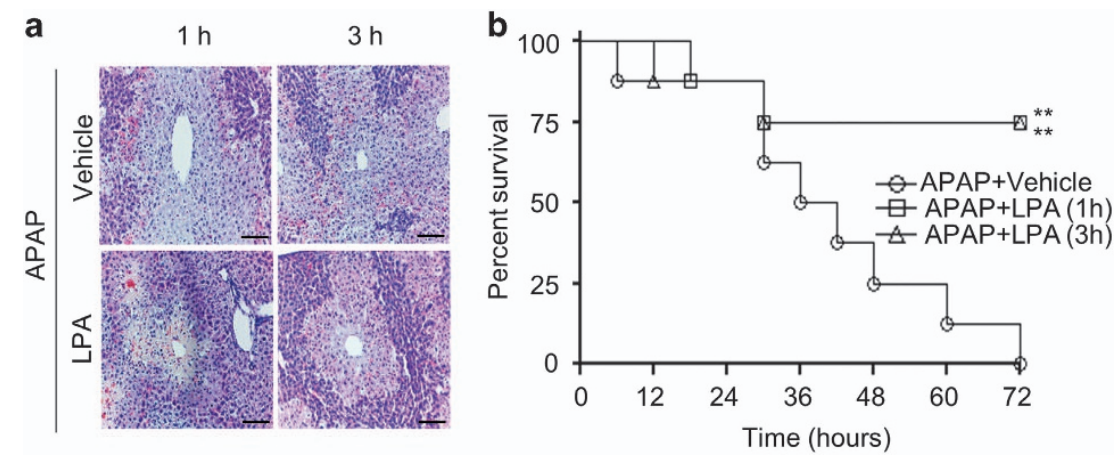

Figure 5 LPA shows therapeutic effects against APAP-induced acute liver injury. (a) After fasting for $16 \mathrm{~h}$, APAP (400 mg kg-1) was administered to mice. Either the vehicle (PBS containing $1 \%$ DMSO) or LPA ( $4 \mathrm{mg} \mathrm{kg}^{-1}$ ) was injected at 1 or $3 \mathrm{~h}$ after the APAP treatment. The mice were killed $12 \mathrm{~h}$ later, and their livers were stained with hematoxylin and eosin (magnification: $\times 200)(\mathbf{a})$. The data are representative of two independent experiments with individual samples. Each experiment was performed in quintuplicates. Scale bar, $100 \mu \mathrm{m}$ (a). (b) For the survival rate, either the vehicle (PBS containing 1\% DMSO) or LPA (4 mg kg-1) was intraperitoneally injected into the mice at 1 or $3 \mathrm{~h}$ after the APAP challenge $\left(750 \mathrm{mg} \mathrm{kg}^{-1}\right)$. The survival rate was monitored every $6 \mathrm{~h}$ for $72 \mathrm{~h}$. ${ }^{*} P<0.01$ by ANOVA. Sample size: $n=8$ (b).

Previous studies have demonstrated that oxidative stress plays a crucial role in hepatotoxicity during the pathogenesis of acute liver injury. ${ }^{24} \mathrm{GSH}$ can remove metabolites of toxic molecules, including NAPQI. ${ }^{25}$ As LPA can protect against acute liver injury, we determined the effect of LPA on the level of GSH, a well-known antioxidant, in a model of acute liver injury. GSH depletion during acute liver injury was significantly recovered by the administration of LPA (Figure 2a). To test the possibility that LPA might have therapeutic effects against APAP by decreasing oxidative stress, we investigated the effect of LPA on the expression of antioxidant genes. We observed that LPA failed to increase the expression levels of several antioxidant genes, including those of $P G C-1 \alpha$, Sod2, Gpx-1 and FGF21 (Figure 2b). These results suggest that LPA administration can increase GSH levels independent of the regulation of some known antioxidant genes. Protein adducts in the liver during drug-induced acute liver injury can impair the function of mitochondria and induce oxidative stress that mediates phosphorylation of GSK3 $\beta$ and JNK. ${ }^{4}$ LPA administration also markedly blocked JNK phosphorylation and subsequent secondary liver damage (Figures $1 \mathrm{a}$ and 2c). Considering previous reports and our results, LPA might be able to inhibit a redox-related pathomechanism that underlies drug overdose-induced acute liver injury, leading to its protective effect against acute liver injury.

Cell surface target receptors play a role in the LPA-induced protective effects against drug-induced acute liver injury. We demonstrated that $\mathrm{LPA}_{1,3,5}$-seletive inhibitors (KI16425 and H2L 5765834) failed to block the effect of LPA (Figure 4). Hepatocytes express several LPA receptors $\left(\mathrm{LPA}_{1,3-6}\right)$ (Figure 4a). Our results suggest that the LPA-induced protective effects against DILI are mediated in a manner that is independent of $\mathrm{LPA}_{1,3,5}$. A molecular target involved in the LPA-induced protective effects against acute liver injury needs to be identified through future experiments. NAC is the only pharmaceutical option that has been available to APAP overdose patients since the 1970 s. ${ }^{21}$ In this study, we showed that
$4 \mathrm{mg} \mathrm{kg}^{-1}$ of LPA resulted in similar preventive effects as $150 \mathrm{mg} \mathrm{kg}^{-1}$ of NAC against APAP-induced mortality (Figure 1e). LPA also showed therapeutic effects to APAPchallenged mice (Figure 5). Therefore, we suggest that LPA and its unidentified target would be considered as novel pharmaceutical targets for APAP overdose patients.

Under acetaminophen-induced acute liver injury, activated hepatic macrophages will release various proinflammatory cytokines, including TNF- $\alpha$ and IL- $1 \beta .^{26}$ These proinflammatory cytokines can enhance inflammation and increase the influx of immune cells, such as monocytes and neutrophils. ${ }^{5}$ In this study, we observed that the administration of LPA markedly decreased these proinflammatory cytokines (TNF- $\alpha$ and IL-1 $\beta$ ) in acute liver injury (Figure 3 ). These results suggest that LPA-induced protective effects against acute liver injury might be mediated by the decreased production of proinflammatory cytokines.

In conclusion, we showed that LPA had protective effects against APAP-induced acute liver injury. Mechanistically, LPA can block hepatocyte death by stimulating GSH recovery independent of the well-known surface receptors $\mathrm{LPA}_{1,3,5}$ in the APAP model. Our results suggest that LPA can be regarded as an important therapeutic agent against drug overdoseinduced acute liver injury.

\section{CONFLICT OF INTEREST}

The authors declare no conflict of interest.

\section{ACKNOWLEDGEMENTS}

This study was supported by a grant from the National Research Foundation of Korea (2015R1A2A1A10054567 (YB)).

\section{PUBLISHER'S NOTE}

This work was supported by the National Research Foundation of Korea(NRF) grants funded by the Korea government(MSIT) (2015R1A2A1A10054567, 2017R1A5A1014560). 
1 Bernal W, Auzinger G, Dhawan A, Wendon J. Acute liver failure. Lancet 2010; 376: 190-201.

2 Cohen SD, Khairallah EA. Selective protein arylation and acetaminopheninduced hepatotoxicity. Drug Metab Rev 1997; 29: 59-77.

3 Knight TR, Kurtz A, Bajt ML, Hinson JA, Jaeschke H. Vascular and hepatocellular peroxynitrite formation during acetaminophen toxicity: role of mitochondrial oxidant stress. Toxicol Sci 2001; 62: 212-220.

4 Han D, Dara L, Win S, Than TA, Yuan L, Abbasi SQ et al. Regulation of drug-induced liver injury by signal transduction pathways: critical role of mitochondria. Trends Pharmacol Sci 2013; 34: 243-253.

5 Karthivashan G, Arulselvan P, Fakurazi S. Pathways involved in acetaminophen hepatotoxicity with specific targets for inhibition/downregulation. RSC Adv 2015; 5: 62040-62051.

6 Marques PE, Amaral SS, Pires DA, Nogueira LL, Soriani FM, Lima BHF et al. Chemokines and mitochondrial products activate neutrophils to amplify organ injury during mouse acute liver failure. Hepatology 2012; 56: 1971-1982.

7 Sevastou I, Kaffe E, Mouratis MA, Aidinis V. Lysoglycerophospholipids in chronic inflammatory disorders: the PLA 2/LPC and ATX/LPA axes. Biochim Biophys Acta 2013; 1831: 42-60.

8 van Corven EJ, Groenink A, Jalink K, Eichholtz T, Moolenaar WH. Lysophosphatidate-induced cell proliferation: Identification and dissection of signaling pathways mediated by G proteins. Cell 1989; 59: 45-54.

9 Yamada T, Yano S, Ogino H, Ikuta K, Kakiuchi S, Hanibuchi M et al. Lysophosphatidic acid stimulates the proliferation and motility of malignant pleural mesothelioma cells through lysophosphatidic acid receptors, LPA1 and LPA2. Cancer Sci 2008; 99: 1603-1610.

10 Willier S, Butt E, Grunewald TGP. Lysophosphatidic acid (LPA) signalling in cell migration and cancer invasion: a focussed review and analysis of LPA receptor gene expression on the basis of more than 1700 cancer microarrays. Biol Cell 2013; 105: 317-333.

11 Kihara Y, Mizuno H, Chun J. Lysophospholipid receptors in drug discovery. Exp Cell Res 2015; 333: 171-177.

12 Choi JW, Herr DR, Noguchi K, Yung YC, Lee CW, Mutoh T et al. LPA receptors: subtypes and biological actions. Annu Rev Pharmacol Toxicol 2010; 50: 157-186.

13 Chou C-H, Lai S-L, Ho C-M, Lin W-H, Chen C-N, Lee P-H et al. Lysophosphatidic acid alters the expression profiles of angiogenic factors, cytokines, and chemokines in mouse liver sinusoidal endothelial cells. PLOS ONE 2015: 10: e0122060.

14 Simo KA, Niemeyer DJ, Hanna EM, Swet JH, Thompson KJ, Sindram D et al. Altered lysophosphatidic acid (LPA) receptor expression during hepatic regeneration in a mouse model of partial hepatectomy. HPB 2014; 16: 534-542.

15 Sautin YY, Crawford JM, Svetlov SI. Enhancement of survival by LPA via Erk1/Erk2 and PI 3-kinase/Akt pathways in a murine hepatocyte cell line. Am J Physiol Cell Physiol 2001; 281: C2010-C2019.

16 Ikeda H, Yatomi Y, Yanase M, Satoh H, Nishihara A, Kawabata M et al. Effects of lysophosphatidic acid on proliferation of stellate cells and hepatocytes in culture. Biochem Biophys Res Commun 1998; 248: 436-440.

17 Watanabe N, Ikeda H, Nakamura K, Ohkawa R, Kume Y, Tomiya T et al. Plasma lysophosphatidic acid level and serum autotaxin activity are increased in liver injury in rats in relation to its severity. Life Sci 2007; 81: $1009-1015$

18 Yu F-X, Zhao B, Panupinthu N, Jewell JL, Lian I, Wang LH et al. Regulation of the Hippo-YAP pathway by G-protein-coupled receptor signaling. Cell 2012; 150: 780-791.

19 David M, Machuca-Gayet I, Kikuta J, Ottewell P, Mima F, Leblanc R et al. Lysophosphatidic acid receptor type 1 (LPA1) plays a functional role in osteoclast differentiation and bone resorption activity. J Biol Chem 2014; 289: 6551-6564.

20 Livak KJ, Schmittgen TD. Analysis of relative gene expression data using real-time quantitative PCR and the 2(-Delta Delta C(T)) Method. Methods 2001; 25: 402-408.

21 Du K, Ramachandran A, Jaeschke H. Oxidative stress during acetaminophen hepatotoxicity: sources, pathophysiological role and therapeutic potential. Redox Biol 2016; 10: 148-156.

22 Jaeschke $H$, McGill MR, Williams CD, Ramachandran A. Current issues with acetaminophen hepatotoxicity-a clinically relevant model to test the efficacy of natural products. Life Sci 2011; 88: 737-745.

23 Gunawan BK, Liu Z-X, Han D, Hanawa N, Gaarde WA, Kaplowitz N. c-Jun $\mathrm{N}$-terminal kinase plays a major role in murine acetaminophen hepatotoxicity. Gastroenterology 2006; 131: 165-178.

24 Jaeschke $H$, Xie Y, McGill MR. Acetaminophen-induced liver injury: from animal models to humans. J Clin Transl Hepatol 2014; 2: 153-161.

25 Jaeschke H, Gores GJ, Cederbaum AI, Hinson JA, Pessayre D, Lemasters JJ. Mechanisms of hepatotoxicity. Toxicol Sci 2002; 65: $166-176$.

26 Krenkel O, Mossanen JC, Tacke F. Immune mechanisms in acetaminophen-induced acute liver failure. Hepatobiliary Surg Nutr 2014; 3: 331-343.

(i) (2) (2) This work is licensed under a Creative Commons Attribution-NonCommercial-ShareAlike 4.0 International License. The images or other third party material in this article are included in the article's Creative Commons license, unless indicated otherwise in the credit line; if the material is not included under the Creative Commons license, users will need to obtain permission from the license holder to reproduce the material. To view a copy of this license, visit http:// creativecommons.org/licenses/by-nc-sa/4.0/

(C) The Author(s) 2017 